PROCEEDINGS OF THE

AMERICAN MATHEMATICAL SOCIETY

Volume 131, Number 6, Pages 1739-1749

S 0002-9939(03)06953-3

Article electronically published on January 15, 2003

\title{
CONVERGENCE RATES OF CASCADE ALGORITHMS
}

\author{
RONG-QING JIA
}

(Communicated by David R. Larson)

AbStRACT. We consider solutions of a refinement equation of the form

$$
\phi=\sum_{\gamma \in \mathbb{Z}^{s}} a(\gamma) \phi(M \cdot-\gamma)
$$

where $a$ is a finitely supported sequence called the refinement mask. Associated with the mask $a$ is a linear operator $Q_{a}$ defined on $L_{p}\left(\mathbb{R}^{s}\right)$ by $Q_{a} \psi:=$ $\sum_{\gamma \in \mathbb{Z}^{s}} a(\gamma) \psi(M \cdot-\gamma)$. This paper is concerned with the convergence of the cascade algorithm associated with $a$, i.e., the convergence of the sequence $\left(Q_{a}^{n} \psi\right)_{n=1,2, \ldots}$ in the $L_{p}$-norm.

Our main result gives estimates for the convergence rate of the cascade algorithm. Let $\phi$ be the normalized solution of the above refinement equation with the dilation matrix $M$ being isotropic. Suppose $\phi$ lies in the Lipschitz space $\operatorname{Lip}\left(\mu, L_{p}\left(\mathbb{R}^{s}\right)\right)$, where $\mu>0$ and $1 \leq p \leq \infty$. Under appropriate conditions on $\psi$, the following estimate will be established:

$$
\left\|Q_{a}^{n} \psi-\phi\right\|_{p} \leq C\left(m^{-1 / s}\right)^{\mu n} \quad \forall n \in \mathbb{N}
$$

where $m:=|\operatorname{det} M|$ and $C$ is a constant. In particular, we confirm a conjecture of A. Ron on convergence of cascade algorithms.

\section{INTRODUCTION}

We are interested in functional equations of the form

$$
\phi=\sum_{\alpha \in \mathbb{Z}^{s}} a(\alpha) \phi(M \cdot-\alpha),
$$

where $\phi$ is the unknown function defined on the $s$-dimensional Euclidean space $\mathbb{R}^{s}$, $a$ is a finitely supported sequence on $\mathbb{Z}^{s}$, and $M$ is an $s \times s$ integer matrix such that $\lim _{n \rightarrow \infty} M^{-n}=0$. The equation (1.1) is called a refinement equation, and the matrix $M$ is called a dilation matrix. Correspondingly, the sequence $a$ is called the refinement mask. Throughout this paper we assume that the mask a satisfies

$$
\sum_{\alpha \in \mathbb{Z}^{s}} a(\alpha)=m:=|\operatorname{det} M|
$$

Under this condition the refinement equation (1.1) has a unique compactly supported distributional solution $\phi$ up to a constant factor.

Received by the editors August 29, 2001.

2000 Mathematics Subject Classification. Primary 39B12, 41A25, 42C40, 65D99.

Key words and phrases. Refinement equations, refinable functions, cascade algorithms, subdivision schemes, rates of convergence.

The author was supported in part by NSERC Canada under Grant OGP 121336. 
For $1 \leq p \leq \infty$, by $L_{p}\left(\mathbb{R}^{s}\right)$ we denote the Banach space of all complex-valued measurable functions $f$ on $\mathbb{R}^{s}$ such that $\|f\|_{p}<\infty$, where

$$
\|f\|_{p}:=\left(\int_{\mathbb{R}^{s}}|f(x)|^{p} d x\right)^{1 / p} \quad \text { for } 1 \leq p<\infty,
$$

and $\|f\|_{\infty}$ is the essential supremum of $|f|$ on $\mathbb{R}^{s}$. By $C\left(\mathbb{R}^{s}\right)$ we denote the space of all continuous functions on $\mathbb{R}^{s}$.

For $1 \leq p \leq \infty$, by $\ell_{p}\left(\mathbb{Z}^{s}\right)$ we denote the Banach space of all complex-valued sequences $b$ on $\mathbb{Z}^{s}$ such that $\|b\|_{p}<\infty$, where

$$
\|b\|_{p}:=\left(\sum_{\alpha \in \mathbb{Z}^{s}}|b(\alpha)|^{p}\right)^{1 / p} \quad \text { for } 1 \leq p<\infty,
$$

and $\|b\|_{\infty}$ is the supremum of $|b|$ on $\mathbb{Z}^{s}$.

Let $\mathbb{N}$ denote the set of positive integers, and let $\mathbb{N}_{0}:=\mathbb{N} \cup\{0\}$. An element of $\mathbb{N}_{0}^{s}$ is called a multi-index. The length of $\mu=\left(\mu_{1}, \ldots, \mu_{s}\right) \in \mathbb{N}_{0}^{s}$ is $|\mu|:=\mu_{1}+\cdots+\mu_{s}$, and the factorial of $\mu$ is $\mu !:=\mu_{1} ! \cdots \mu_{s} !$. For $\mu=\left(\mu_{1}, \ldots, \mu_{s}\right) \in \mathbb{N}_{0}^{s}$ and $x=$ $\left(x_{1}, \ldots, x_{s}\right) \in \mathbb{R}^{s}$, define

$$
x^{\mu}:=x_{1}^{\mu_{1}} \cdots x_{s}^{\mu_{s}} .
$$

The function $x \mapsto x^{\mu}\left(x \in \mathbb{R}^{s}\right)$ is called a monomial and its (total) degree is $|\mu|$. A polynomial is a linear combination of monomials. The degree of a polynomial $q=\sum_{\mu} c_{\mu} x^{\mu}$ is defined to be $\operatorname{deg} q:=\max \left\{|\mu|: c_{\mu} \neq 0\right\}$. By $\Pi_{k}$ we denote the linear space of all polynomials of degree at most $k$.

For a vector $y=\left(y_{1}, \ldots, y_{s}\right) \in \mathbb{R}^{s}$, its norm is defined as $|y|:=\left|y_{1}\right|+\cdots+\left|y_{s}\right|$. We use $D_{y}$ to denote the differential operator given by

$$
D_{y} f(x):=\lim _{t \rightarrow 0} \frac{f(x+t y)-f(x)}{t}, \quad x \in \mathbb{R}^{s} .
$$

Moreover, we use $\nabla_{y}$ to denote the difference operator given by

$$
\nabla_{y} f=f-f(\cdot-y) .
$$

Let $e_{1}, \ldots, e_{s}$ be the unit coordinate vectors in $\mathbb{R}^{s}$. For $j=1, \ldots, s$, we write $D_{j}$ for $D_{e_{j}}$. For a multi-index $\mu=\left(\mu_{1}, \ldots, \mu_{s}\right), D^{\mu}$ stands for the differential operator $D_{1}^{\mu_{1}} \cdots D_{s}^{\mu_{s}}$. Given a polynomial $q=\sum_{\mu} c_{\mu} x^{\mu}$, we use $q(D)$ to denote the differential operator $\sum_{\mu} c_{\mu} D^{\mu}$.

For a positive integer $k$ and $1 \leq p \leq \infty$, the Sobolev seminorm $|\cdot|_{k, p}$ is defined as

$$
|f|_{k, p}:=\sum_{|\mu|=k}\left\|D^{\mu} f\right\|_{p}
$$

The Sobolev space $W_{p}^{k}\left(\mathbb{R}^{s}\right)$ consists of all functions $f \in L_{p}\left(\mathbb{R}^{s}\right)$ such that $|f|_{k, p}<$ $\infty$.

Lipschitz spaces are defined in terms of the modulus of smoothness. The modulus of continuity of a function $f$ in $L_{p}\left(\mathbb{R}^{s}\right)$ is defined by

$$
\omega(f, h)_{p}:=\sup _{|y| \leq h}\left\|\nabla_{y} f\right\|_{p}, \quad h \geq 0 .
$$

Let $k$ be a positive integer. The $k$ th modulus of smoothness of $f \in L_{p}\left(\mathbb{R}^{s}\right)$ is defined by

$$
\omega_{k}(f, h)_{p}:=\sup _{|y| \leq h}\left\|\nabla_{y}^{k} f\right\|_{p}, \quad h \geq 0 .
$$


For $1 \leq p \leq \infty$ and $0<\nu \leq 1$, the Lipschitz space $\operatorname{Lip}\left(\nu, L_{p}\left(\mathbb{R}^{s}\right)\right)$ consists of all functions $f \in L_{p}\left(\mathbb{R}^{s}\right)$ for which

$$
\omega(f, h)_{p} \leq C h^{\nu} \quad \forall h>0,
$$

where $C$ is a positive constant independent of $h$. For $\nu>0$ we write $\nu=r+\eta$, where $r$ is an integer and $0<\eta \leq 1$. The Lipschitz $\operatorname{space} \operatorname{Lip}\left(\nu, L_{p}\left(\mathbb{R}^{s}\right)\right)$ consists of those functions $f \in L_{p}\left(\mathbb{R}^{s}\right)$ for which $D^{\mu} f \in \operatorname{Lip}\left(\eta, L_{p}\left(\mathbb{R}^{s}\right)\right)$ for all multi-indices $\mu$ with $|\mu|=r$.

The Fourier transform of a function $f \in L_{1}\left(\mathbb{R}^{s}\right)$ is defined by

$$
\hat{f}(\omega):=\int_{\mathbb{R}^{s}} f(x) e^{-i x \cdot \omega} d x, \quad \omega \in \mathbb{R}^{s},
$$

where $i$ stands for the imaginary unit, and $x \cdot \omega$ denotes the inner product of two vectors $x$ and $\omega$ in $\mathbb{R}^{s}$. The Fourier transform can be extended to the class of tempered distributions (see [1]). For instance, the Fourier transform of the Dirac distribution $\delta$ is 1 , while the Fourier transform of a polynomial $q$ is $(2 \pi)^{s} q(i D) \delta$.

Let $Q_{a}$ be the cascade operator given by

$$
Q_{a} f:=\sum_{\alpha \in \mathbb{Z}^{s}} a(\alpha) f(M \cdot-\alpha), \quad f \in L_{p}\left(\mathbb{R}^{s}\right) .
$$

For $n=1,2, \ldots$, we have (see [7])

$$
Q_{a}^{n} f=\sum_{\alpha \in \mathbb{Z}^{s}} a_{n}(\alpha) f\left(M^{n} \cdot-\alpha\right)
$$

where the sequences $a_{n}$ are given by

$$
a_{1}=a \quad \text { and } \quad a_{n}(\alpha)=\sum_{\beta \in \mathbb{Z}^{s}} a(\alpha-M \beta) a_{n-1}(\beta), \quad \alpha \in \mathbb{Z}^{s} .
$$

The iteration scheme $f_{n}:=Q_{a}^{n} f(n=0,1,2, \ldots)$ is called a cascade algorithm. The main purpose of this paper is to investigate rates of convergence of cascade algorithms.

Convergence of cascade algorithms are closely related to approximation with shift-invariant spaces. Let $f$ be a compactly supported integrable function on $\mathbb{R}^{s}$. We say that $f$ satisfies the Strang-Fix conditions of order $k$ (see [18]) if

$$
\hat{f}(0)=1 \quad \text { and } \quad D^{\mu} \hat{f}(2 \pi \beta)=0 \quad \forall|\mu|<k \quad \text { and } \quad \beta \in \mathbb{Z}^{s} .
$$

If $f$ satisfies the Strang-Fix conditions of order $k$, then the shift-invariant space generated by $f$ provides approximation order $k$.

Let $\phi$ be the unique compactly supported distributional solution of the refinement equation (1.1) subject to the condition $\hat{\phi}(0)=1$. Such a solution is called the normalized solution. If the normalized solution $\phi$ lies in $L_{p}\left(\mathbb{R}^{s}\right)(1 \leq p \leq \infty)$, then it was shown in [13] that $\phi$ satisfies the Strang-Fix conditions of order 1. Suppose $\psi$ is a compactly supported function in $L_{p}\left(\mathbb{R}^{s}\right)(1 \leq p \leq \infty)$. If the cascade algorithm with the initial function $\psi$ converges to $\phi$ in the $L_{p}$ norm, that is,

$$
\lim _{n \rightarrow \infty}\left\|Q_{a}^{n} \psi-\phi\right\|_{p}=0
$$


then it was shown in 7] that $\psi$ satisfies the Strang-Fix conditions of order 1. A typical choice of the initial function is the tensor product of the hat functions:

$$
\chi(x):=\prod_{j=1}^{s} \max \left\{1-\left|x_{j}\right|, 0\right\}, \quad x=\left(x_{1}, \ldots, x_{s}\right) \in \mathbb{R}^{s} .
$$

If $\lim _{n \rightarrow \infty}\left\|Q_{a}^{n} \chi-\phi\right\|_{p}=0$, then $\lim _{n \rightarrow \infty}\left\|Q_{a}^{n} \psi-\phi\right\|_{p}=0$ for any compactly supported function $\psi$ in $L_{p}\left(\mathbb{R}^{s}\right)$ satisfying the Strang-Fix conditions of order 1 (see [7]). However, the sequence $\left(Q_{a}^{n} \chi\right)_{n=1,2, \ldots}$ may diverge, even if the normalized solution $\phi$ lies in $L_{p}\left(\mathbb{R}^{s}\right)$. Thus it is desirable to find appropriate conditions on $\psi$ such that $Q_{a}^{n} \psi$ converges to $\phi$ in the $L_{p}$ norm.

For a compactly supported distribution $f$ on $\mathbb{R}^{s}$, we use $K(f)$ to denote the linear space of all sequences $c$ on $\mathbb{Z}^{s}$ such that

$$
\sum_{\alpha \in \mathbb{Z}^{s}} c(\alpha) f(\cdot-\alpha)=0 .
$$

If $K(f)=\{0\}$, then we say that the (multi-integer) shifts of $f$ are linearly independent. It was shown in [16] that the shifts of $f$ are linearly independent if and only if for any $\zeta \in \mathbb{C}^{s}$ there exists some $\beta \in \mathbb{Z}^{s}$ such that $\hat{f}(\zeta+2 \pi \beta) \neq 0$.

In Section 4 we will establish the following result:

Theorem 1.1. Suppose the normalized solution $\phi$ of the refinement equation (1.1) lies in $L_{p}\left(\mathbb{R}^{s}\right), 1 \leq p<\infty$. If $\psi$ is a compactly supported function in $L_{p}\left(\mathbb{R}^{s}\right)$ satisfying the Strang-Fix conditions of order 1, and if $K(\phi) \subseteq K(\psi)$, then

$$
\lim _{n \rightarrow \infty}\left\|Q_{a}^{n} \psi-\phi\right\|_{p}=0 .
$$

In the case $p=\infty$, if both $\phi$ and $\psi$ are continuous, then $Q_{a}^{n} \psi$ converges to $\phi$ uniformly.

In particular, if the shifts of $\phi$ are linearly independent, then the cascade sequence $Q_{a}^{n} \psi$ converges to $\phi$ for any compactly supported function $\psi$ in $L_{p}\left(\mathbb{R}^{s}\right)$ satisfying the Strang-Fix conditions of order 1 . This result is also valid, provided the shifts of $\phi$ are stable.

Let $f$ be a compactly supported function in $L_{p}\left(\mathbb{R}^{s}\right)(1 \leq p \leq \infty)$. We say that the (multi-integer) shifts of $f$ are stable, if there exist two positive constants $C_{1}$ and $C_{2}$ such that

$$
C_{1}\|b\|_{p} \leq\left\|\sum_{\alpha \in \mathbb{Z}^{s}} b(\alpha) f(\cdot-\alpha)\right\|_{p} \leq C_{2}\|b\|_{p} \quad \forall b \in \ell_{p}\left(\mathbb{Z}^{s}\right) .
$$

The shifts of $f$ are stable if and only if for any $\xi \in \mathbb{R}^{s}$ there exists some $\beta \in \mathbb{Z}^{s}$ such that $\hat{\phi}(\xi+2 \pi \beta) \neq 0$ (see [14]). Consequently, linear independence implies stability.

Convergence rates of subdivision schemes were studied in [5] and [2] for box splines. This problem was investigated in [3, 6] and [20] for dyadic refinable functions. Also, see 4 and [22] for some related work. Suppose $\phi$ is the normalized solution of the dyadic refinement equation

$$
\phi=\sum_{\alpha \in \mathbb{Z}^{s}} a(\alpha) \phi(2 \cdot-\alpha),
$$

where $a$ is finitely supported and $\sum_{\alpha \in \mathbb{Z}^{s}} a(\alpha)=2^{s}$. If $\phi$ lies in the Sobolev space $W_{\infty}^{k}\left(\mathbb{R}^{s}\right)$, then $\phi$ satisfies the Strang-Fix conditions of order $k$ (see [3]). If, in 
addition, the shifts of $\phi$ are stable, then

$$
\left\|Q_{a}^{n} \psi-\phi\right\|_{\infty} \leq C 2^{-k n} \quad \forall n \in \mathbb{N}
$$

for any compactly supported continuous function $\psi$ on $\mathbb{R}^{s}$ satisfying the condition

$$
D^{\mu} \hat{\psi}(2 \pi \beta)=D^{\mu} \hat{\phi}(2 \pi \beta) \quad \forall \beta \in \mathbb{Z}^{s} \text { and }|\mu|<k .
$$

This result was established in [21].

Recently, Ron [17] conjectured that the same result is also true under the condition $K(\phi) \subseteq K(\psi)$ instead of stability of $\phi$. In fact, his conjecture was stated for Sobolev spaces $W_{p}^{\alpha}\left(\mathbb{R}^{s}\right)$ of fractional order. In [19] Sun gave a partial answer to the conjecture. Ron's conjecture will be confirmed in this paper.

Our result will be valid for the more general refinement equation (1.1). We assume that the dilation matrix $M$ is isotropic, that is, $M$ is similar to a diagonal matrix $\operatorname{diag}\left\{\sigma_{1}, \ldots, \sigma_{s}\right\}$ with $\left|\sigma_{1}\right|=\cdots=\left|\sigma_{s}\right|=m^{1 / s}$, where $m=|\operatorname{det} M|$.

Theorem 1.2. Let $\phi$ be the normalized solution of the refinement equation (1.1) with the dilation matrix $M$ being isotropic. Suppose $\phi \in \operatorname{Lip}\left(\mu, L_{p}\left(\mathbb{R}^{s}\right)\right)$, where $\mu>0$ and $1 \leq p \leq \infty$. Let $k$ be the integer such that $k-1<\mu \leq k$. Let $\psi$ be a compactly supported function in $L_{p}\left(\mathbb{R}^{s}\right)$ satisfying the Strang-Fix conditions of order $k$. If $K(\phi) \subseteq K(\psi)$, and if $D^{\nu} \hat{\psi}(0)=D^{\nu} \hat{\phi}(0)$ for all $|\nu|<k$, then there exists a positive constant $C$ such that

$$
\left\|Q_{a}^{n} \psi-\phi\right\|_{p} \leq C\left(m^{-1 / s}\right)^{\mu n} \quad \forall n \in \mathbb{N} .
$$

The proof of this theorem will be completed in Section 4. Preparatory to the proof, we shall investigate relative stability in Section 2 and approximation schemes in Section 3.

\section{Relative Stability}

Let $\ell\left(\mathbb{Z}^{s}\right)$ be the linear space of all sequences on $\mathbb{Z}^{s}$, and let $\ell_{0}\left(\mathbb{Z}^{s}\right)$ be the linear space of all finitely supported sequences on $\mathbb{Z}^{s}$. For $u \in \ell\left(\mathbb{Z}^{s}\right)$ and $v \in \ell_{0}\left(\mathbb{Z}^{s}\right)$, define

$$
\langle u, v\rangle:=\sum_{\alpha \in \mathbb{Z}^{s}} u(\alpha) v(-\alpha) .
$$

With respect to this bilinear form, $\ell\left(\mathbb{Z}^{s}\right)$ is the algebraic dual of $\ell_{0}\left(\mathbb{Z}^{s}\right)$.

If $\langle u, v\rangle=0$, then $u$ and $v$ are orthogonal and we write $u \perp v$. For a linear subspace $V$ of $\ell_{0}\left(\mathbb{Z}^{s}\right)$, we define

$$
V^{\perp}:=\left\{u \in \ell\left(\mathbb{Z}^{s}\right):\langle u, v\rangle=0 \forall v \in V\right\} .
$$

If $w \in \ell_{0}\left(\mathbb{Z}^{s}\right)$ satisfies $w \perp V^{\perp}$, then $w \in V$. This claim can be justified by contraposition. Suppose $w \notin V$. Let $W$ be the linear span of $V$ and $w$. Then we can find a linear functional $f$ on $W$ such that $f$ vanishes on $V$ and $f(w)=1$. This linear functional can be extended to a linear functional on $\ell_{0}\left(\mathbb{Z}^{s}\right)$. Since $\ell\left(\mathbb{Z}^{s}\right)$ is the algebraic dual of $\ell_{0}\left(\mathbb{Z}^{s}\right)$, this means that there exists some element $u$ in $\ell\left(\mathbb{Z}^{s}\right)$ such that $u \perp V$ and $\langle u, w\rangle=1$. This contradicts the assumption $w \perp V^{\perp}$.

The main result of this section is the following lemma on relative stability.

Lemma 2.1. Let $\phi$ and $\psi$ be compactly supported functions in $L_{p}\left(\mathbb{R}^{s}\right)(1 \leq p \leq \infty)$. Suppose $K(\phi) \subseteq K(\psi)$. Then there exists a positive constant $C$ such that

$$
\left\|\sum_{\alpha \in \mathbb{Z}^{s}} b(\alpha) \psi(\cdot-\alpha)\right\|_{L_{p}\left(\mathbb{R}^{s}\right)} \leq C\left\|\sum_{\alpha \in \mathbb{Z}^{s}} b(\alpha) \phi(\cdot-\alpha)\right\|_{L_{p}\left(\mathbb{R}^{s}\right)} \quad \forall b \in \ell_{p}\left(\mathbb{Z}^{s}\right) .
$$


Proof. We can find linearly independent functions $\phi_{1}, \ldots, \phi_{m}$ in $L_{p}\left(\mathbb{R}^{s}\right)$ supported on $[0,1]^{s}$ such that

$$
\phi=\sum_{j=1}^{m} \sum_{\beta \in \mathbb{Z}^{s}} \phi_{j}(\cdot-\beta) \lambda_{j}(\beta),
$$

where $\lambda_{1}, \ldots, \lambda_{m} \in \ell_{0}\left(\mathbb{Z}^{s}\right)$ (see [10]). For $b \in \ell_{p}\left(\mathbb{Z}^{s}\right)$, it follows that

$$
\sum_{\alpha \in \mathbb{Z}^{s}} b(\alpha) \phi(\cdot-\alpha)=\sum_{j=1}^{m} \sum_{\gamma \in \mathbb{Z}^{s}}\left(b * \lambda_{j}\right)(\gamma) \phi_{j}(\cdot-\gamma),
$$

where $b * \lambda$ denotes the convolution of $b$ and $\lambda$. Since the functions $\phi_{1}, \ldots, \phi_{m}$ are supported on $[0,1]^{s}$ and are linearly independent, there exists a constant $C_{1}$ such that

$$
\left\|b * \lambda_{j}\right\|_{p} \leq C_{1}\left\|\sum_{\alpha \in \mathbb{Z}^{s}} b(\alpha) \phi(\cdot-\alpha)\right\|_{p}, \quad j=1, \ldots, m .
$$

Let $V_{\phi}$ be the linear span of (multi-integer) shifts of $\lambda_{1}, \ldots, \lambda_{m}$. In light of (2.2) we see that $u \in K(\phi)$ if and only if $u$ is orthogonal to shifts of $\lambda_{1}, \ldots, \lambda_{m}$. Consequently, $K(\phi)=V_{\phi}^{\perp}$.

Similarly, we can find linearly independent functions $\psi_{1}, \ldots, \psi_{n}$ in $L_{p}\left(\mathbb{R}^{s}\right)$ supported on $[0,1]^{s}$ such that

$$
\psi=\sum_{k=1}^{n} \sum_{\beta \in \mathbb{Z}^{s}} \psi_{k}(\cdot-\beta) \mu_{k}(\beta),
$$

where $\mu_{1}, \ldots, \mu_{n} \in \ell_{0}\left(\mathbb{Z}^{s}\right)$. We have $\mu_{k} \perp K(\psi)$ for $k=1, \ldots, n$. By the assumption, $K(\phi) \subseteq K(\psi)$. Hence, $\mu_{k} \perp K(\phi)=V_{\phi}^{\perp}$. By the remark made at the beginning of this section, we assert that $\mu_{k}$ lies in $V_{\phi}$. In other words, each $\mu_{k}$ can be expressed as

$$
\mu_{k}=\sum_{j=1}^{m} c_{k j} * \lambda_{j}
$$

where $c_{k j} \in \ell_{0}\left(\mathbb{Z}^{s}\right), j=1, \ldots, m, k=1, \ldots, n$. Note that

$$
\sum_{\alpha \in \mathbb{Z}^{s}} b(\alpha) \psi(\cdot-\alpha)=\sum_{k=1}^{n} \sum_{\gamma \in \mathbb{Z}^{s}}\left(b * \mu_{k}\right)(\gamma) \psi_{k}(\cdot-\gamma) .
$$

Hence, there exists a constant $C_{2}$ such that

$$
\left\|\sum_{\alpha \in \mathbb{Z}^{s}} b(\alpha) \psi(\cdot-\alpha)\right\|_{p} \leq C_{2} \sum_{k=1}^{n}\left\|b * \mu_{k}\right\|_{p}
$$

But

$$
\left\|b * \mu_{k}\right\|_{p}=\left\|\sum_{j=1}^{n} c_{k j} *\left(\lambda_{j} * b\right)\right\|_{p} \leq \sum_{j=1}^{n}\left\|c_{k j}\right\|_{1}\left\|b * \lambda_{j}\right\|_{p}, \quad k=1, \ldots, n .
$$

This in combination with (2.3) and (2.4) gives the desired result (2.1). 


\section{Approximation sChemes}

In this section we discuss approximation schemes induced by quasi-projection operators.

For $f \in L_{p}\left(\mathbb{R}^{s}\right)$ and $g \in L_{p^{\prime}}\left(\mathbb{R}^{s}\right)\left(1 / p^{\prime}+1 / p=1\right)$ we define

$$
\langle f, g\rangle:=\int_{\mathbb{R}^{s}} f(y) g(-y) d y .
$$

Let $\phi$ be a compactly supported function in $L_{p}\left(\mathbb{R}^{s}\right)$, and let $g$ be a compactly supported function in $L_{p^{\prime}}\left(\mathbb{R}^{s}\right)$, where $1 / p^{\prime}+1 / p=1$. Consider the linear operator $P$ on $L_{p}\left(\mathbb{R}^{s}\right)$ defined by

$$
P f=\sum_{\alpha \in \mathbb{Z}^{s}}\langle f, g(\cdot-\alpha)\rangle \phi(\cdot-\alpha), \quad f \in L_{p}\left(\mathbb{R}^{s}\right) .
$$

Such an operator is called a quasi-projection operator. Clearly, $P$ is a bounded operator on $L_{p}\left(\mathbb{R}^{s}\right)$. For $h>0$, let

$$
P^{h} f:=\sum_{\alpha \in \mathbb{Z}^{s}}\left\langle f, h^{-1} g(\cdot / h-\alpha)\right\rangle \phi(\cdot / h-\alpha), \quad f \in L_{p}\left(\mathbb{R}^{s}\right) .
$$

It is easily seen that $\left\|P^{h}\right\|=\|P\|$.

Approximation by means of quasi-projection operators is discussed in [15]. In particular, the following result was established there: If the quasi-projection operator $P$ reproduces all polynomials of degree at most $k-1$, i.e., $P q=q$ for all $q \in \Pi_{k-1}$, then there exists a constant $C>0$ such that

$$
\left\|P^{h} f-f\right\|_{p} \leq C h^{k}|f|_{k, p} \quad \forall f \in W_{p}^{k}\left(\mathbb{R}^{s}\right) \text { and } h>0 .
$$

In [12] the above result was extended to the following form: Under the same conditions as above, there exists a constant $C>0$ such that

$$
\left\|P^{h} f-f\right\|_{p} \leq C \omega_{k}(f, h)_{p} \quad \forall h>0
$$

for $f \in L_{p}\left(\mathbb{R}^{s}\right)\left(f \in C\left(\mathbb{R}^{s}\right)\right.$ in the case $\left.p=\infty\right)$.

Now let us discuss approximation with shift-invariant spaces scaled by a dilation matrix. Let $M$ be an isotropic dilation matrix with $m:=|\operatorname{det} M|$. For $n=1,2, \ldots$, let $P_{n}$ be the quasi-projection operator given by

$$
P_{n} f:=\sum_{\alpha \in \mathbb{Z}^{s}}\left\langle f, m^{n} g\left(M^{n} \cdot-\alpha\right)\right\rangle \phi\left(M^{n} \cdot-\alpha\right), \quad f \in L_{p}\left(\mathbb{R}^{s}\right) .
$$

Note that the absolute value of every eigenvalue of the matrix $M$ is equal to $\mathrm{m}^{1 / \mathrm{s}}$. We have the following result, whose proof can be found in [12].

Lemma 3.1. Let $P$ be the quasi-projection operator given in (3.1). If $P q=q$ for all $q \in \Pi_{k-1}$, then there exists a constant $C>0$ such that

$$
\left\|P_{n} f-f\right\|_{p} \leq C \omega_{k}\left(f, m^{-n / s}\right)_{p}
$$

for $n=1,2, \ldots$ and $f \in L_{p}\left(\mathbb{R}^{s}\right) \quad\left(f \in C\left(\mathbb{R}^{s}\right)\right.$ in the case $\left.p=\infty\right)$. In particular, if $f \in \operatorname{Lip}\left(\mu, L_{p}\left(\mathbb{R}^{s}\right)\right)$, where $\mu>0$ and $1 \leq p \leq \infty$, then

$$
\left\|P_{n} f-f\right\|_{p} \leq C\left(m^{-1 / s}\right)^{\mu n} \text {. }
$$

Consequently, if the quasi-projection operator $P$ reproduces constants, i.e., $P 1=1$, then

$$
\lim _{n \rightarrow \infty}\left\|P_{n} f-f\right\|_{p}=0
$$


for $f \in L_{p}\left(\mathbb{R}^{s}\right)(1 \leq p<\infty)$, or $f \in C\left(\mathbb{R}^{s}\right)(p=\infty)$. In fact, in order for (3.3) to be true, the dilation matrix $M$ does not need to be isotropic.

The following lemma gives a sufficient condition for the quasi-projection operator $P$ to reproduce all polynomials of degree at most $k-1$.

Lemma 3.2. Suppose $\phi$ satisfies the Strang-Fix conditions of order $k$. Let $g$ be a compactly supported function in $L_{p^{\prime}}\left(\mathbb{R}^{s}\right), 1 / p^{\prime}+1 / p=1$. If $D^{\nu}(1-\hat{g} \hat{\phi})(0)=0$ for all $|\nu|<k$, then the quasi-projection operator $P$ given in (3.1) reproduces all polynomials of degree at most $k-1$, i.e., $P q=q$ for all $q \in \Pi_{k-1}$.

Proof. Let $q \in \Pi_{k-1}$. We observe that $P q=q$ if and only if

$$
\sum_{\alpha \in \mathbb{Z}^{s}} r(\alpha) \phi(\cdot-\alpha)=q,
$$

where $r:=q * g$ is a polynomial. By using the Poisson summation formula, it was proved in [8] that

$$
\sum_{\alpha \in \mathbb{Z}^{s}} r(\alpha) \phi(\cdot-\alpha)=\hat{\phi}(-i D) r .
$$

The Fourier transform of $\hat{\phi}(-i D) r$ is $\hat{\phi} \hat{r}=\hat{\phi} \hat{g}\left((2 \pi)^{s} \hat{q}(i D) \delta\right)$, where $\delta$ stands for the Dirac distribution. Consequently, $\hat{\phi}(-i D) r=q$ if and only if $\hat{\phi} \hat{g}(\hat{q}(i D) \delta)=\hat{q}(i D) \delta$. This happens if

$$
D^{\nu}(1-\hat{g} \hat{\phi})(0)=0 \quad \forall|\nu|<k .
$$

Hence, (3.4) implies $P q=q$ for all $q \in \Pi_{k-1}$. The proof of the lemma is complete.

For given $c_{\nu}(|\nu|<k)$ we can find a compactly supported function $g \in L_{p^{\prime}}\left(\mathbb{R}^{s}\right)$ such that $D^{\nu} \hat{g}(0)=c_{\nu}$ for all $|\nu|<k$. Therefore, there exists a compactly supported function $g$ in $L_{p^{\prime}}\left(\mathbb{R}^{s}\right)$ such that (3.4) is true.

\section{CAScade Algorithms}

We are in a position to prove Theorems 1.1 and 1.2. Our proofs follow the lines of $[9]$.

Proof of Theorem 1.1. We only consider the case $1 \leq p<\infty$. The proof for the case $p=\infty$ is similar.

Since the normalized solution $\phi$ of (1.1) lies in $L_{p}\left(\mathbb{R}^{s}\right), \phi$ must satisfy the StrangFix conditions of order 1 (see [13]). Let $g$ be a compactly supported function in $L_{p^{\prime}}\left(\mathbb{R}^{s}\right)\left(1 / p^{\prime}+1 / p=1\right)$ such that $\hat{g}(0)=1$. According to our assumption, $\psi$ satisfies the Strang-Fix conditions of order 1. By Lemma 3.2 the quasi-projection operators $P_{\phi}$ and $P_{\psi}$ given by

$$
P_{\phi} f=\sum_{\alpha \in \mathbb{Z}^{s}}\langle f, g(\cdot-\alpha)\rangle \phi(\cdot-\alpha) \quad \text { and } \quad P_{\psi} f=\sum_{\alpha \in \mathbb{Z}^{s}}\langle f, g(\cdot-\alpha)\rangle \psi(\cdot-\alpha)
$$

reproduce constants, i.e., $P_{\phi} 1=1$ and $P_{\psi} 1=1$. For $n=1,2, \ldots$, let

$$
f_{n}:=\sum_{\alpha \in \mathbb{Z}^{s}} b_{n}(\alpha) \phi\left(M^{n} \cdot-\alpha\right) \quad \text { and } \quad g_{n}:=\sum_{\alpha \in \mathbb{Z}^{s}} b_{n}(\alpha) \psi\left(M^{n} \cdot-\alpha\right),
$$

where

$$
b_{n}(\alpha)=\left\langle\phi, m^{n} g\left(M^{n} \cdot-\alpha\right)\right\rangle, \quad \alpha \in \mathbb{Z}^{s} .
$$


By (3.3) we have

$$
\lim _{n \rightarrow \infty}\left\|f_{n}-\phi\right\|_{p}=0 \quad \text { and } \quad \lim _{n \rightarrow \infty}\left\|g_{n}-\phi\right\|_{p} \rightarrow 0 .
$$

Let

$$
h_{n}:=Q_{a}^{n} \psi=\sum_{\alpha \in \mathbb{Z}^{s}} a_{n}(\alpha) \psi\left(M^{n} \cdot-\alpha\right),
$$

where the sequences $a_{n}(n=1,2, \ldots)$ are given in (1.2). Clearly,

$$
\phi=\sum_{\alpha \in \mathbb{Z}^{s}} a_{n}(\alpha) \phi\left(M^{n} \cdot-\alpha\right) .
$$

It follows that

$$
\left(f_{n}-\phi\right)\left(M^{-n} x\right)=\sum_{\alpha \in \mathbb{Z}^{s}}\left(b_{n}-a_{n}\right)(\alpha) \phi(x-\alpha), \quad x \in \mathbb{R}^{s} .
$$

Similarly,

$$
\left(g_{n}-h_{n}\right)\left(M^{-n} x\right)=\sum_{\alpha \in \mathbb{Z}^{s}}\left(b_{n}-a_{n}\right)(\alpha) \psi(x-\alpha), \quad x \in \mathbb{R}^{s} .
$$

Since $K(\phi) \subseteq K(\psi)$, Lemma 2.1 tells us that there exists a positive constant $C$ independent of $n$ such that

$$
\left\|\left(g_{n}-h_{n}\right)\left(M^{-n} \cdot\right)\right\|_{p} \leq C\left\|\left(f_{n}-\phi\right)\left(M^{-n} \cdot\right)\right\|_{p} .
$$

Consequently,

$$
\left\|g_{n}-h_{n}\right\|_{p} \leq C\left\|f_{n}-\phi\right\|_{p} .
$$

But

$$
\left\|h_{n}-\phi\right\|_{p} \leq\left\|h_{n}-g_{n}\right\|_{p}+\left\|g_{n}-\phi\right\|_{p} \leq C\left\|f_{n}-\phi\right\|_{p}+\left\|g_{n}-\phi\right\|_{p} .
$$

This in connection with (4.2) shows that $\lim _{n \rightarrow \infty}\left\|h_{n}-\phi\right\|_{p}=0$, as desired.

Proof of Theorem 1.2. Since the normalized solution $\phi$ of (1.1) lies in the Sobolev space $W_{p}^{k-1}\left(\mathbb{R}^{s}\right), \phi$ must satisfy the Strang-Fix conditions of order $k$ (see [3] and [11]). We can find a compactly supported function $g \in L_{p^{\prime}}\left(\mathbb{R}^{s}\right)\left(1 / p^{\prime}+1 / p=1\right)$ such that (3.4) is valid. By our assumption, $D^{\nu} \hat{\psi}(0)=D^{\nu} \hat{\phi}(0)$ for all $|\nu|<k$. Hence, we also have

$$
D^{\nu}(1-\hat{g} \hat{\psi})(0)=0 \quad \forall|\nu|<k .
$$

By Lemma 3.2, the quasi-projection operators $P_{\phi}$ and $P_{\psi}$ given in (4.1) reproduce all polynomials of order at most $k-1$, i.e., $P_{\phi} q=P_{\psi} q=q$ for all $q \in \Pi_{k-1}$.

Let $a_{n}$ and $b_{n}(n=1,2, \ldots)$ be the same sequences as above. Let

$$
f_{n}:=\sum_{\alpha \in \mathbb{Z}^{s}} b_{n}(\alpha) \phi\left(M^{n} \cdot-\alpha\right), \quad g_{n}:=\sum_{\alpha \in \mathbb{Z}^{s}} b_{n}(\alpha) \psi\left(M^{n} \cdot-\alpha\right)
$$

and

$$
h_{n}:=Q_{a}^{n} \psi=\sum_{\alpha \in \mathbb{Z}^{s}} a_{n}(\alpha) \psi\left(M^{n} \cdot-\alpha\right) .
$$

Since $\phi \in \operatorname{Lip}\left(\mu, L_{p}\left(\mathbb{R}^{s}\right)\right)$, (3.2) tells us that there exists a positive constant $C_{1}$ independent of $n$ such that

$$
\left\|f_{n}-\phi\right\|_{p} \leq C_{1}\left(m^{-1 / s}\right)^{\mu n} \text { and }\left\|g_{n}-\phi\right\|_{p} \leq C_{1}\left(m^{-1 / s}\right)^{\mu n} .
$$

Note that $\phi=Q_{a}^{n} \phi$. Hence, it follows that

$$
\left\|\sum_{\alpha \in \mathbb{Z}^{s}}\left[b_{n}(\alpha)-a_{n}(\alpha)\right] \phi\left(M^{n} \cdot-\alpha\right)\right\|_{p} \leq C_{1}\left(m^{-1 / s}\right)^{\mu n} .
$$


Since $K(\phi) \subseteq K(\psi)$, by Lemma 2.1, there exists a positive constant $C_{2}$ such that

$$
\left\|\sum_{\alpha \in \mathbb{Z}^{s}}\left[b_{n}(\alpha)-a_{n}(\alpha)\right] \psi\left(M^{n} \cdot-\alpha\right)\right\|_{p} \leq C_{2}\left(m^{-1 / s}\right)^{\mu n}
$$

that is,

$$
\left\|g_{n}-h_{n}\right\|_{p} \leq C_{2}\left(m^{-1 / s}\right)^{\mu n}
$$

Therefore,

$$
\left\|\phi-h_{n}\right\|_{p} \leq\left\|\phi-g_{n}\right\|_{p}+\left\|g_{n}-h_{n}\right\|_{p} \leq C\left(m^{-1 / s}\right)^{\mu n},
$$

where $C:=C_{1}+C_{2}$. The proof of Theorem 1.2 is complete.

\section{REFERENCES}

1. J. Barros-Neto, An Introduction to the Theory of Distributions, Marcel Dekker, New York, 1973. MR 57:1113

2. C. de Boor, K. Höllig, and S. Riemenschneider, Box Splines, Springer-Verlag, New York, 1993. MR 94k:65004

3. A. S. Cavaretta, W. Dahmen, and C. A. Micchelli, Stationary Subdivision, Memoirs of Amer. Math. Soc., Volume 93, 1991. MR 92h:65017

4. D. R. Chen, R. Q. Jia, and S. D. Riemenschneider, Convergence of vector subdivision schemes in Sobolev spaces, Applied and Computational Harmonic Analysis 12 (2002), 128-149. MR 2002k:65220

5. W. Dahmen, N. Dyn, and D. Levin, On the convergence rates of the subdivision algorithms for box spline surfaces, Constr. Approx. 1 (1985), 305-332. MR 88h:41016

6. S. Durand, Convergence of cascade algorithms introduced by I. Daubechies, Numer. Algorithms 4 (1993), 307-322. MR 94i:65019

7. B. Han and R. Q. Jia, Multivariate refinement equations and convergence of subdivision schemes, SIAM J. Math. Anal. 29 (1998), 1177-1199. MR 99f:41018

8. R. Q. Jia, A dual basis for the integer translates of an exponential box spline, Rocky Mountain J. Math. 23 (1993), 223-242. MR 94a:41022

9. R. Q. Jia, Subdivision schemes in $L_{p}$ spaces, Advances in Comp. Math. 3 (1995), 309-341. MR 96d:65028

10. R. Q. Jia, Shift-invariant spaces and linear operator equations, Israel J. Math. 103 (1998), 259-288. MR 99d:41016

11. R. Q. Jia, Approximation properties of multivariate wavelets, Math. Comp. 67 (1998), 647665. MR 98g:41020

12. R. Q. Jia, Approximation with scaled shift-invariant spaces by means of quasi-projection operators, manuscript.

13. R. Q. Jia and C. A. Micchelli, Using the refinement equation for the construction of prewavelets II: Powers of two, Curves and Surfaces (P. J. Laurent, A. Le Méhauté, and L. L. Schumaker, eds.), Academic Press, New York, 1991, pp. 209-246. MR 93e:65024

14. R. Q. Jia and C. A. Micchelli, On linear independence of integer translates of a finite number of functions, Proc. Edinburgh Math. Soc. 36 (1992), 69-85. MR 94e:41044

15. J. J. Lei, R. Q. Jia, and E. W. Cheney, Approximation from shift-invariant spaces by integral operators, SIAM J. Math. Anal. 28 (1997), 481-498. MR 98h:41026

16. A. Ron, A necessary and sufficient condition for the linear independence of the integer translates of a compactly supported distribution, Constr. Approx. 5 (1989), 297-308. MR 90g:41019

17. A. Ron, Wavelets and their associated operators, in Approximation Theory IX, Vol. 2: Computational Aspects, C. K. Chui and L. L. Schumaker (eds.), pp. 283-317, Vanderbilt University, 1998. MR 2000k:41003

18. G. Strang and G. Fix, A Fourier analysis of the finite-element variational method, in Constructive Aspects of Functional Analysis, G. Geymonat (ed.), C.I.M.E. (1973), pp. 793-840.

19. Q. Y. Sun, Convergence of cascade algorithms and smoothness of refinable distributions, manuscript.

20. L. F. Villemoes, Wavelet analysis of refinement equations, SIAM J. Math. Anal. 25 (1994), 1433-1460. MR 96f:39009 
21. S. R. Zhang, Refinable Functions and Subdivision Schemes, Ph.D. Thesis, University of Alberta, 1998.

22. D. X. Zhou, Norms concerning subdivision sequences and their applications in wavelets, Applied and Computational Harmonic Analysis 11 (2001), 329-346. MR 2002i:42055

Department of Mathematics, University of Alberta, Edmonton, Alberta, Canada T6G $2 \mathrm{G} 1$

E-mail address: jia@xihu.math.ualberta.ca 